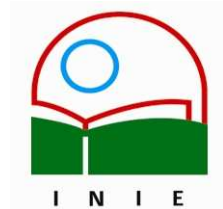

Actualidades Investigativas en Educación

Revista Electrónica publicada por el

Instituto de Investigación en Educación

Universidad de Costa Rica

ISSN 1409-4703

http://revista.inie.ucr.ac.cr

COSTA RICA

\title{
EL PAPEL DE LAS COMPETENCIAS INDIVIDUALES Y COLECTIVAS EN LOS SISTEMAS DE ACCIÓN
}

THE ROLE OF INDIVIDUAL AND COLLECTIVE COMPETENCES IN ACTION SYSTEMS

\author{
Volumen 9, Número 2 \\ pp. 1-19
}

Este número se publicó el 30 de agosto 2009

Juan B. Climént Bonilla

La revista está indexada en los directorios:

LATINDEX, REDALYC, IRESIE, $\underline{\text { CLASE}}, \underline{\text { DIALNET, DOAJ, E-REVIST@S, }}$

La revista está incluida en los sitios:

REDIE, RINACE, OEI, MAESTROTECA, PREAL, HUASCARAN, CLASCO

Los contenidos de este artículo están bajo una licencia Creative Commons

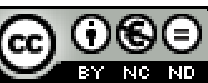




\title{
EL PAPEL DE LAS COMPETENCIAS INDIVIDUALES Y COLECTIVAS EN LOS SISTEMAS DE ACCIÓN
}

\author{
THE ROLE OF INDIVIDUAL AND COLLECTIVE COMPETENCES IN ACTION SYSTEMS
}

\author{
Juan B. Climént Bonilla
}

\begin{abstract}
Resumen: El principal propósito de este artículo, producto de un extensa revisión de términos y conceptos relacionados con educación y capacitación basadas en competencias, es aclarar el papel de las competencias individuales y colectivas en el contexto de los sistemas de acción, donde ambos tipos se complementan -como instrumentos de la formación de individuos y grupos- en la solución de problemas y necesidades que afectan a las personas en distintos espacios de participación e intervención social. Con el mismo propósito, se examinan varias de las diferencias y puntos de encuentro, entre competencias individuales y colectivas, especialmente en lo que concierne a los componentes primarios y las funciones sustantivas de los sistemas de acción. Finalmente, se advierte sobre los efectos adversos que pueden tener las competencias falsas o desnaturalizadas, en el funcionamiento y la sustentabilidad de dichos sistemas.
\end{abstract}

Palabras clave: COMPETENCIAS INDIVIDUALES, COMPETENCIAS COLECTIVAS, SISTEMA DE ACCIÓN, TIPOLOGÍA DE LAS COMPETENCIAS

\begin{abstract}
The main purpose of this article, product of an extensive revision of terms and concepts related to competence-based education and training, is to clarify the role of individual and collective competences in the context of action systems, where both types complement each other -as individual and group professional development instruments - in the solution of problems and needs that affect individuals in various spaces of social participation and intervention. With the same purpose, several differences and communalities, between individual and collective competences, are examined, specially in relation to action systems' primary components and substantive functions. Finally, it is made notice about the adverse effects that false or denaturalized competences may have in such systems functioning and sustainability.
\end{abstract}

Key words: INDIVIDUAL COMPETENCES, COLLECTIVE COMPETENCES, ACTION SYSTEM, TYPOLOGY OF COMPETENCES

\section{INTRODUCCIÓN}

El desarrollo de las nuevas tecnologías de la información y la comunicación, ha dado paso a un creciente despliegue de cambios en la manera de ver y hacer las cosas, en millones de personas alrededor del mundo. En torno a los procesos globalizadores han surgido diversos conceptos — como por ejemplo: era de la informática, sociedad del conocimiento, aprendizaje a lo largo de la vida y educación basada en competencias (Argüelles, 1996; OIT, 1997; OCDE, 1997)—, ampliamente difundidos entre sociedades, organizaciones e individuos cuya

\footnotetext{
${ }^{1}$ Doctor en Educación, por la Universidad de Cornell; ha participado como consultor en diversos estudios y proyectos en materia de desarrollo rural y educación para adultos; desde 1986, es profesor investigador de la Universidad Autónoma Metropolitana, Unidad Xochimilco, en la ciudad de México.
}

Dirección electrónica: jcliment@correo.xoc.uam.mx

Artículo recibido: 29 de mayo, 2009

Aprobado: 24 de agosto, 2009 
actividad y desarrollo — político, económico, comercial, social, cultural - en gran medida se basan en el acceso a la información y el conocimiento a través de sistemas computarizados. Evidentemente, el mundo de la educación, lejos de permanecer al margen de dichos procesos, aparece al centro tanto de sus efectos como de sus causas.

Ante la necesidad de elevar la competitividad económica (Burton, 1992; Lloyd y Cook, 1993), varios países han optado por una amplia conversión de la oferta educativa -particularmente en instituciones de educación media superior y superior, y en centros de capacitación-, de modalidades "tradicionales" a esquemas de educación basados en estándares de competencia para la certificación individual (Coles y Werquin, 2007). Bajo este enfoque, hoy día existe una extensa variedad de tipos y subtipos de competencias, que atañen tanto a las diferentes etapas de educación (básica, media y superior), como a las distintas rutas y espacios de aprendizaje (formal, no formal e informal); sin embargo, en general, las competencias se valoran y reconocen a nivel individual (Lloyd y Cook, 1993, p. 17; OCDE, 1997; Nyatanga, Forman y Fox, 1998), y no en la esfera de grupos humanos (equipos de trabajo, organizaciones, sociedades), donde sus connotaciones y alcances son distintos, considerando el carácter y la magnitud de los problemas y necesidades a resolver, la complejidad de los contextos en que las competencias se adquieren y aplican, y la cantidad y variedad de los agentes sociales involucrados.

Para analizar las diferencias entre competencias individuales y colectivas, y ahondar sobre su trascendencia y relación, el presente artículo asume la existencia de un continuo entre unas y otras. Este continuo discurre en doble sentido: por un lado, de la esfera personal —en que las competencias individuales se gestan, a través del aprendizaje y la experiencia acumulados en el curso de la vida-, a los sistemas de acción —en que las competencias individuales se complementan entre sí, con la participación conjunta de diversos actores y roles, para dar lugar a competencias colectivas-; y por otro, en dirección opuesta, de los sistemas de acción —propios de organizaciones y estructuras sociales complejas-, a la esfera personal —de modo que las competencias colectivas propician la articulación de las competencias individuales, y con ello, el mejor ejercicio y desarrollo de éstas. 


\section{EL CONCEPTO DE COMPETENCIA CENTRADO EN EL INDIVIDUO}

Aunque el fomento de las competencias en oficios y profesiones se remonta muchos siglos atrás, no es sino hasta décadas recientes en que este esquema se adopta dentro de grandes sistemas organizacionales que operan como instrumentos de política educativa y laboral, en el plano de estrategias competitivas a escala mundial. Por ello, el significado del término competencia es determinante en el carácter de políticas e iniciativas de educación y empleo, que tienen a las competencias en el centro de sus objetivos y lineamientos. A continuación aparecen cinco definiciones que proporcionan una clara idea de lo que se quiere decir y se puede entender por competencia, en la corriente dominante de la formación bajo este enfoque:

a) Capacidad de satisfacer demandas o llevar a cabo tareas con éxito, constituida de dimensiones cognitivas y no cognitivas (OCDE, 2002, p. 7).

b) Dimensión de conductas abiertas y manifiestas que le permiten a una persona rendir eficientemente (Woodruffe, 1993; citado por Rodríguez, s.f.).

c) Habilidad para desempeñar actividades al nivel esperado en el empleo (Lloyd y Cook, 1993, p. 14).

d) Característica subyacente de un individuo, causalmente relacionada con un rendimiento efectivo o superior en una situación o trabajo, definido bajo ciertos criterios (Spencer y Spencer, 1993; citados por Rodríguez, s.f.).

e) Estándares que especifican el nivel de conocimientos y habilidades necesarios para realizar con éxito, en el mundo laboral, funciones adecuadas para cada grupo ocupacional (Comisión Europea, s.f., a y b).

Las cinco definiciones enfatizan la capacidad de los individuos para alcanzar resultados favorables o deseados, especialmente - se infiere - en lo que concierne a las expectativas de terceros. En las dos primeras el contexto de las demandas y el rendimiento queda abierto, puede ser de orden académico, profesional o laboral, entre otros; en tanto que para las tres siguientes, explícitamente se refiere a los requerimientos del mercado de trabajo. De esta forma, en lo que pudiera tenerse como una "ecuación" de la competencia, las cinco definiciones destacan el interés por los resultados. Al respecto, cabe agregar, las dos últimas definiciones, de las cinco enunciadas, se circunscriben a estándares de competencia laboral; es decir, a referentes (criterios y evidencias) de las expectativas de desempeño de un individuo en determinada actividad o función productiva. 
Más allá de las diferencias y similitudes señaladas, las cinco definiciones comparten, de manera implícita, una concepción de las competencias en el ámbito individual, por encima del colectivo; ninguna hace referencia explícita a las competencias a nivel de grupos; en gran medida se apegan al modelo convencional de las competencias individuales (véase figura 1). Esta noción es válida en la implementación de esquemas estandarizados de educación y capacitación, donde predomina el enfoque individualizado, pero no para entender el contexto y papel de las competencias en entidades sociales complejas - por ejemplo, una o más sociedades en determinada región o regiones del mundo-, como es el caso de los sistemas de acción.

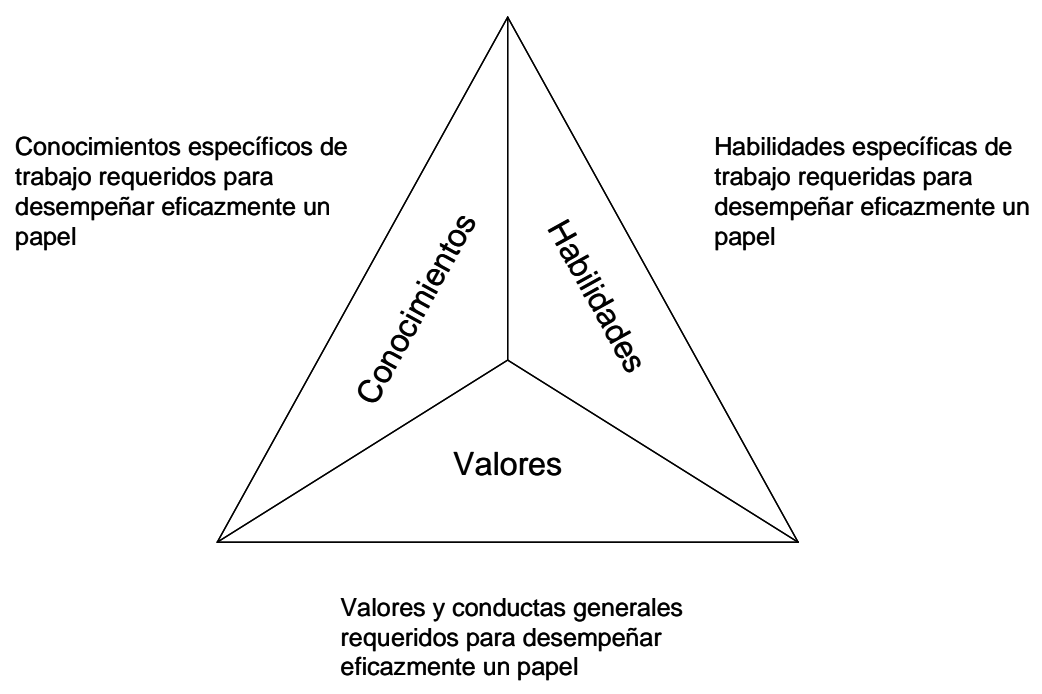

Figura 1. Modelo del perfil de la competencia. Tomado de Kaplan y Norton (2004, p. 232).

Debido a que en la literatura especializada se utilizan indistintamente los conceptos de "educación basada en normas (estándares) de competencia" (Morfín, 1996, p. 81), y de "educación basada en competencias" (Gonczi, 1996, p. 265), pudiera pensarse que hablar de estándares de competencia o, simplemente, de competencias, es prácticamente lo mismo; no obstante, conviene aclarar, los estándares de competencia son instrumentos de evaluación del desempeño individual en tareas o funciones de especial interés; las competencias, en cambio, podemos considerarlas como instrumentalidades (cualidades instrumentales) de la formación de las personas, forjadas a lo largo de la vida, en el ámbito individual y de la interacción con otros. Las competencias, por naturaleza, exceden las 
nociones de aprendizaje y desempeño estandarizadas; de ahí que, valga subrayar, una cosa son los estándares de competencia, y otra, mucho más compleja, las competencias que poseemos las personas para desenvolvernos en la vida, como individuos y grupos.

\section{TIPOLOGÍA DE LAS COMPETENCIAS}

En la figura 2 aparecen algunos de los tipos de competencias más difundidos en la literatura sobre el tema. Como puede apreciarse, los tipos de competencias están agrupados, bajo una estructura común, en cuatro estratos: a) bases, b) procesos, c) sistemas y d) telos. En seguida se hace una descripción sucinta de cada uno de ellos, para ubicar su relación y papel dentro de dicha estructura.

Bases:

- Competencias transcurriculares. -Abarcan un amplio espectro de conocimientos, habilidades y valores que son fundamentales para el adecuado desenvolvimiento personal, social y laboral de los individuos; se aprenden sobre todo en el transcurso de la educación inicial, desde la infancia y la adolescencia temprana, hasta la madurez (CERI, 1997, p. 18).

- Aprendizajes centrales. -Procesos primarios de aprendizaje, de la mayor importancia durante la niñez, la adolescencia y la juventud, a partir de los cuales, y en gran medida, se forja la capacidad de aprender, el aprendizaje profundo, las metacompetencias y el aprendizaje a lo largo de la vida (CERI, 2000, p. 22).

- Competencias socio-normativas. -Revisten gran importancia para la vida y el adecuado funcionamiento de los individuos en la sociedad, especialmente en lo relativo a la educación social y cívica (CERI, 1997, pp. 19-22).

- Competencias técnico-instrumentales. -Como las socio-normativas, son relevantes a la vida y el adecuado funcionamiento de los individuos en la sociedad. Se les considera fundamentales para el aprendizaje de todas y cada una de las materias y disciplinas que se imparten en los diferentes grados, niveles y modalidades de la educación inicial; y asimismo, trascienden en el aprendizaje extraescolar, el desempeño laboral y la vida social (CERI, 1997, pp. 19-22).

- Competencias claves. -Son importantes en múltiples aspectos de la vida, contribuyen al éxito de las personas y a que una sociedad funcione bien (OCDE, 2002, p. 9). 
- Intrapersonales. - Se trata de habilidades, destrezas y actitudes que determinan la conducta individual, las reacciones, los estados mentales, el estilo de imitación y el estilo de comunicación (Salovey y Mayer, 1990, p. 189).

- Interpersonales. -Tienen un carácter eminentemente social; atañen a la interacción con otros y la colaboración (Tuning Project, 2004).

\section{Procesos:}

- Competencias laborales. - Implicadas en el desempeño satisfactorio de las tareas y actividades de un puesto de trabajo o función productiva. El marco de estándares de normalización y certificación, de este tipo de competencias, corre a cargo de los organismos responsables del diseño y elaboración de normas de competencia dentro de cierta jurisdicción- para diferentes ocupaciones, industrias y sectores productivos.

- Competencias genéricas o transferibles. —Describen comportamientos asociados con desempeños comunes a diversas ocupaciones y ramas de actividad productiva (CINTERFOR/OIT, s.f.).

- Competencias específicas o técnicas. -Operan en el contexto de funciones o actividades productivas, y están asociadas a ocupaciones particulares (CONOCER, 1996).

\section{Sistemas:}

- Competencias directivas o gerenciales. -Asociadas con las habilidades de liderazgo que permiten, a uno o más individuos, mejorar las prácticas necesarias para que una empresa pueda desempeñar sus funciones y misión de la manera más eficiente posible (Air Force Personnel Center, s.f.).

- Competencias sistémicas. -Conciernen a los sistemas en su conjunto (Tuning Project, 2004).

Telos:

- Metacompetencias. — Se refieren a competencias de orden superior —más allá de la información básica y las habilidades rutinarias- derivadas de procesos de interiorización y aprendizaje profundo, como la creatividad, el sentido común, el pensamiento crítico, la capacidad reflexiva, el análisis sistémico u holista, la agilidad mental, el conocimiento de sí mismo y el aprendizaje balanceado de distintas competencias (Buckley, Monks y McKevitt, 2002, p. 5). 


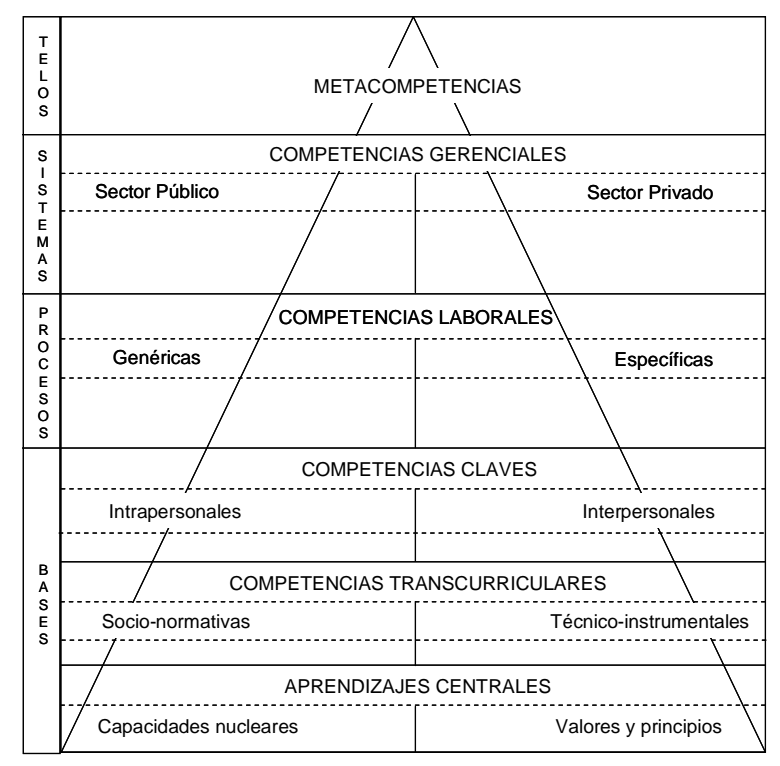

Figura 2. Distintos tipos de competencias.

Desde una perspectiva conjunta, puede decirse que los valores y las capacidades de aprendizaje individual, en su condición de aprendizajes centrales, sirven de apoyo y sostén para las competencias adquiridas en el curso de la vida; por lo que, a falta de estos valores y capacidades, el sentido de las competencias se debilita o desvirtúa. Las competencias claves y las competencias transcurriculares - que en muchos casos sería mejor llamar habilidades, en lugar de competencias - también son parte de las bases de la formación individual. Ambos tipos de competencias se yuxtaponen, al punto de manejarlas sin distinción. No obstante, mientras las competencias claves están abiertas al aprendizaje formal, no formal e informal, a lo largo de la vida, las transcurriculares -que en sí son claves - se circunscriben a la educación inicial: desde el nivel básico hasta el superior. Las competencias laborales se ubican en el ámbito de procesos productivos, en tanto las competencias gerenciales o directivas lo hacen en el plano de sistemas que atañen a intereses organizacionales y colectivos de distinta naturaleza y nivel. Finalmente, en el estrato más alto se encuentran las metacompetencias, en estrecha interrelación con los demás estratos y, particularmente, con las capacidades de aprendizaje y los valores para la vida y el trabajo. 
Es interesante observar que todos los tipos y subtipos de competencias descritos, en clara concomitancia con las definiciones de competencia proporcionadas en el apartado anterior, confieren al término significados y representaciones en la esfera de lo individual. En ningún caso aluden expresamente a las competencias a nivel colectivo, es decir, en el contexto de grupos de personas - con diversos roles, habilidades y perfiles de formación, para afrontar problemas y necesidades comunes-, pese a que este contexto es fundamental para explicar el papel de las competencias, en general: tanto en lo individual como en lo colectivo. Esta distinción es importante por dos grandes razones: primero, una cosa son las competencias de cada individuo -en el terreno de equipos de trabajo, organizaciones, sectores productivos o fuerzas laborales-, y otra, lo que las personas mismas, en conjunto, pueden y consiguen hacer; y segundo, el fomento de las competencias individuales y colectivas adquiere sentido y fortaleza en la medida en que existe reciprocidad y congruencia entre las competencias de los individuos y las del grupo -o grupos- a que éstos pertenecen. Asimismo, tal distinción puede considerarse intrascendente o inoperante para fines de evaluación de competencias; sin embargo, es crítica cuando se trata de mejorar la formación de competencias en entidades sociales de distinto tamaño y género, como son: equipos de trabajo, organizaciones, sectores productivos, gremios profesionales, comunidades y sociedades.

\section{COMPETENCIAS INDIVIDUALES Y COMPETENCIAS COLECTIVAS}

La concepción de las competencias como instrumentalidades (cualidades instrumentales) de la formación (individual y colectiva) para la solución de problemas complejos — propios de la realidad, o las realidades, en que las personas viven y se forjan-, permite la asociación de competencias individuales y colectivas en un mismo esquema. De tal modo, el alcance de las competencias puede dimensionarse en esferas de intervención que van de lo individual a lo colectivo y, viceversa, de lo colectivo a lo individual.

Necesariamente, la solución de un problema implica un modelo (tácito o declarado) que anticipe el proceso de solución, a manera de guía (ex ante), o se desprenda de éste, como producto (ex post). En ambos casos, las competencias (individuales, grupales, organizativas) involucradas en la solución de un problema, guardan estrecha correspondencia con el proceso/modelo de solución (adoptado o generado). Los problemas, las soluciones y las competencias son interdependientes, no pueden entenderse si no es entre sí. Es en este Volumen 9, Número 2, Año 2009, ISSN 1409-4703 
contexto en que las competencias - como tales - son comprensibles más allá de sus elementos formales —estándares, conocimientos, habilidades, destrezas, capacidades, actitudes y valores-y el convencional énfasis en la individualidad.

\section{Competencias individuales}

Cabría definirlas como las instrumentalidades de la formación de un individuo, que trascienden positivamente en el desarrollo y desenvolvimiento personal, social y laboral de éste, bajo determinadas circunstancias y contextos. Girard (2004) circunscribe el concepto de competencias individuales en tres dominios: el de las competencias claves, el de las competencias profesionales y el de la interacción entre ambas, como se aprecia en la siguiente figura.

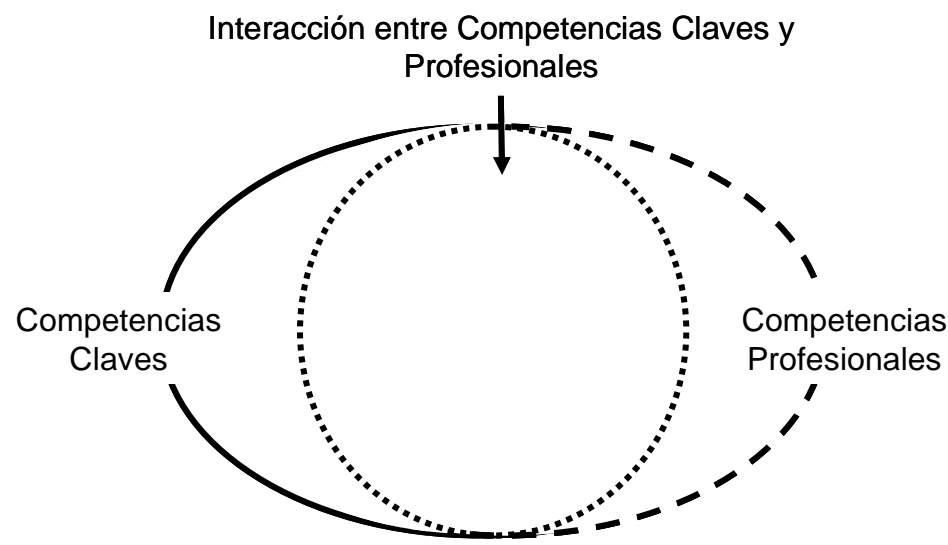

Figura 3. Dimensiones de las competencias individuales. Adaptado de Girard (2004, p. 5).

Desde la perspectiva de formación a lo largo de la vida, las competencias individuales son el resultado de un prolongado proceso de aprendizaje: primero de habilidades esenciales (claves, básicas, académicas), durante la niñez y la juventud; luego de competencias genéricas (claves, básicas, académicas, vocacionales), en la preparación de jóvenes y adultos para la vida y el trabajo; y, posteriormente, en el desarrollo de competencias profesionales/laborales (genéricas, específicas, sistémicas), para responder a necesidades concretas de formación y empleo. Véase figura 4. 


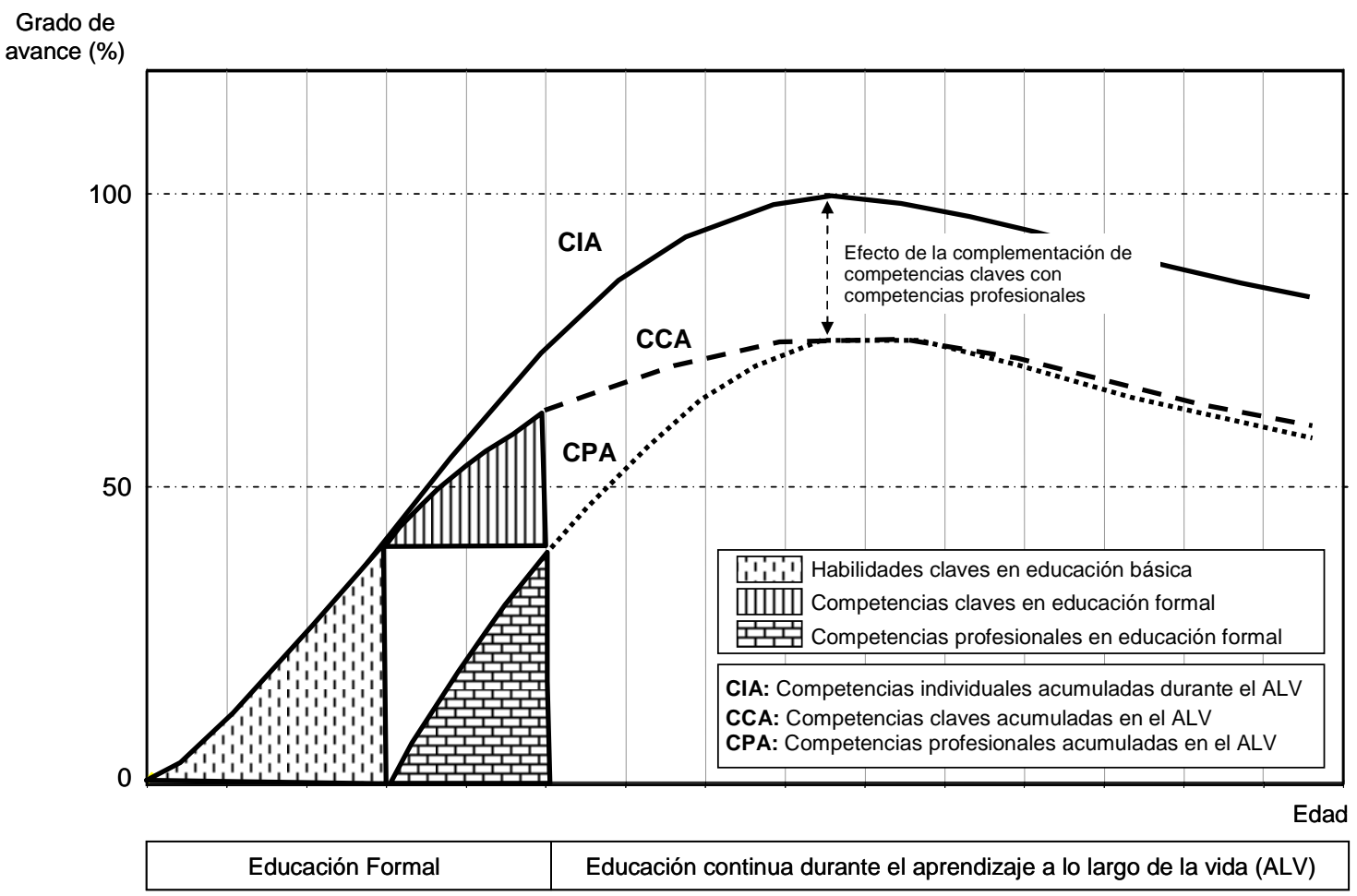

Figura 4. Adquisición y desarrollo de competencias a lo largo de la vida (caso hipotético). Adaptado de Girard (2004, p. 5).

Usualmente, los enfoques de educación y capacitación basados en competencias se estructuran mediante estándares (criterios y evidencias) de desempeño, que constituyen como se mencionó anteriormente- instrumentos de evaluación individualizada. En esta tendencia, los modelos representados en las figuras 3 y 4 , como los de las figuras anteriores, abordan las competencias en el ámbito individual, al margen del colectivo. En consecuencia, puede incurrirse en la desatención — deliberada o no- de la naturaleza y alcances de las competencias colectivas, en detrimento de las capacidades de uno o más grupos de personas para prevenir y resolver problemas de su interés y responsabilidad. Generalmente las competencias individuales conducen, pero no equivalen, a las competencias colectivas.

\section{Competencias colectivas}

Como extensión de las competencias individuales, y en el marco de la definición proporcionada en el apartado anterior, se trata de las instrumetalidades de la formación que 
permiten a un grupo de personas (equipo de trabajo, organización, comunidad, actividad laboral, sector productivo, sociedad) alcanzar una comprensión conjunta de los problemas que les conciernen -incluyendo diferencias y conflictos entre las partes involucradas-, de modo que puedan participar activa, coordinada y eficientemente en su solución - a partir de objetivos y esfuerzos compartidos-, desde las esferas de responsabilidad y los espacios de intervención que a cada quien corresponden.

La competencia colectiva busca la complementariedad y el encadenamiento de competencias. La puesta en práctica de las competencias de cada actor está relacionada con la competencia de los demás actores de la cadena. Sólo en la medida en que cada individuo encuentra competencias complementarias a las suyas, podrá poner estas últimas plenamente en práctica (Fernández-Salinero, 2006, p. 144).

Así, el significado y alcance de las competencias colectivas se desprende de numerosos factores, como por ejemplo:

- Contexto socio-cultural donde estas competencias se adquieren y aplican.

- Perfiles de formación (habilidades, conocimientos, valores) de los actores (líderes, grupos, sectores de la población) involucrados.

- Complejidad y diversidad de los problemas y necesidades a resolver.

- Desarrollo científico, tecnológico, económico y social del sistema humano de acción, en conjunto.

- Brechas en materia de educación, género, salud, tecnología, productividad y niveles de vida.

- Esquemas (normas, intereses, valores, oportunidades) de participación política, económica y social.

- Cultura de valores (respeto, colaboración, honestidad, eficiencia, bien común, trabajo en equipo).

\section{SISTEMAS DE ACCIÓN}

Antes de abordar este punto, es pertinente resaltar la trascendencia de la obra de Talcott Parsons, que es central al conocimiento de los sistemas de acción, como se verá más adelante. 
Nadie entre los contemporáneos ha desarrollado una teoría comparable de la sociedad ...La obra actualmente presente no tiene competencia en cuanto a la altura de abstracción y diferenciación, alcance teórico social y sistemática, con simultánea conexión con la literatura de diferentes áreas de investigación. No obstante de que el interés en esta teoría haya disminuido desde mediados de los años 60 y que el planteamiento de Parsons haya sido empujado hacia el trasfondo momentáneamente por parte de planteamientos de investigación hermenéuticos y orientados hacia la crítica, actualmente no se puede tomar en serio ninguna teoría social que no, por lo menos, se refiera a la de Parsons (Habermas, 1981, citado por Jokisch, s.f.).

Las competencias individuales y colectivas son inherentes al sentido y los atributos de los sistemas de acción; entendidos éstos, como "estructuras y procesos por medio de los que los seres humanos constituyen intenciones significativas y con mayor o menor éxito, las aplican en situaciones concretas" (Parsons, 1974, p. 15). Por consiguiente, el papel de las competencias, como instrumentos de la formación para la solución de problemas, es consustancial a las características y propiedades de las estructuras y los procesos que conforman tales sistemas.

Según Parsons (1974), el sistema humano de acción comprende cuatro elementos primarios: a) organismos conductuales, b) sistemas de personalidad c) sistemas culturales, y d) sistemas sociales; y cuatro funciones primarias: a) adaptación, b) alcance de metas c) mantenimiento de patrones, y $d$ ) integración. De tal modo, estos elementos y funciones constituyen las bases estructurales y funcionales del sistema de acción (Parsons, 1974):

- Organismo conductual. Como sitio de las capacidades humanas primarias, que son el sostén de los otros sistemas, le concierne la función de adaptación. Incluye un conjunto de condiciones a las que la acción debe adaptarse, y comprende el mecanismo primario de interrelación con el medio físico. Se basa en los atributos del sistema nervioso y la actividad motora para enfrentarse al medio ambiente.

- Sistema de personalidad. Es la "agencia" primordial de los procesos de acción y, por lo tanto, también de la aplicación de requisitos y principios culturales. La personalidad de los individuos tiene supremacía en el alcance de metas.

- Sistema cultural. Tiene prioridad sobre el mantenimiento y el cambio creativo de patrones de acción. 
- Sistema social. Se ocupa de la integración del sistema de acción, a través de la coordinación de los distintos actores.

A continuación, el modelo de la figura 5, basado en el concepto de Parsons de un sistema de acción, remite las competencias individuales y colectivas al ámbito y la interrelación de los cuatro sistemas señalados. De la naturaleza de estos sistemas — considerados en el modelo subsistemas del sistema de acción- se desprende igual número de funciones centrales, y de éstas, a la vez, los principales roles de los agentes sociales involucrados, para cada sistema y el sistema de acción, en general.

En esencia, el modelo muestra cuatro grandes subsistemas estrechamente vinculados, que en conjunto constituyen un suprasistema donde las personas, como individuos y grupos, desarrollan un universo común — de alguna manera compartido— en la interacción de sus espacios de actividad. Es importante señalar que las competencias de las personas, a nivel individual y de grupos, están unidas al andamiaje del sistema de acción, pero más importante aún, es el hecho de que dichas competencias son fundamentales tanto en la construcción de este sistema, como en la conformación de las características y atributos que determinan su sustentabilidad.

El subsistema organismo conductual es eminentemente individual, a diferencia del subsistema social, cuya naturaleza refiere a la condición de grupo. Así, las acciones cobran distintas dimensiones a través de los cuatro subsistemas, de lo individual a lo colectivo, y viceversa, de lo colectivo a lo individual, como las hondas del agua en la superficie de un estanque. La acción en el ámbito individual (organismo conductual y de personalidad) puede trascender al ámbito colectivo (cultural y social), como también, la acción en el ámbito colectivo repercutir en el ámbito individual. 


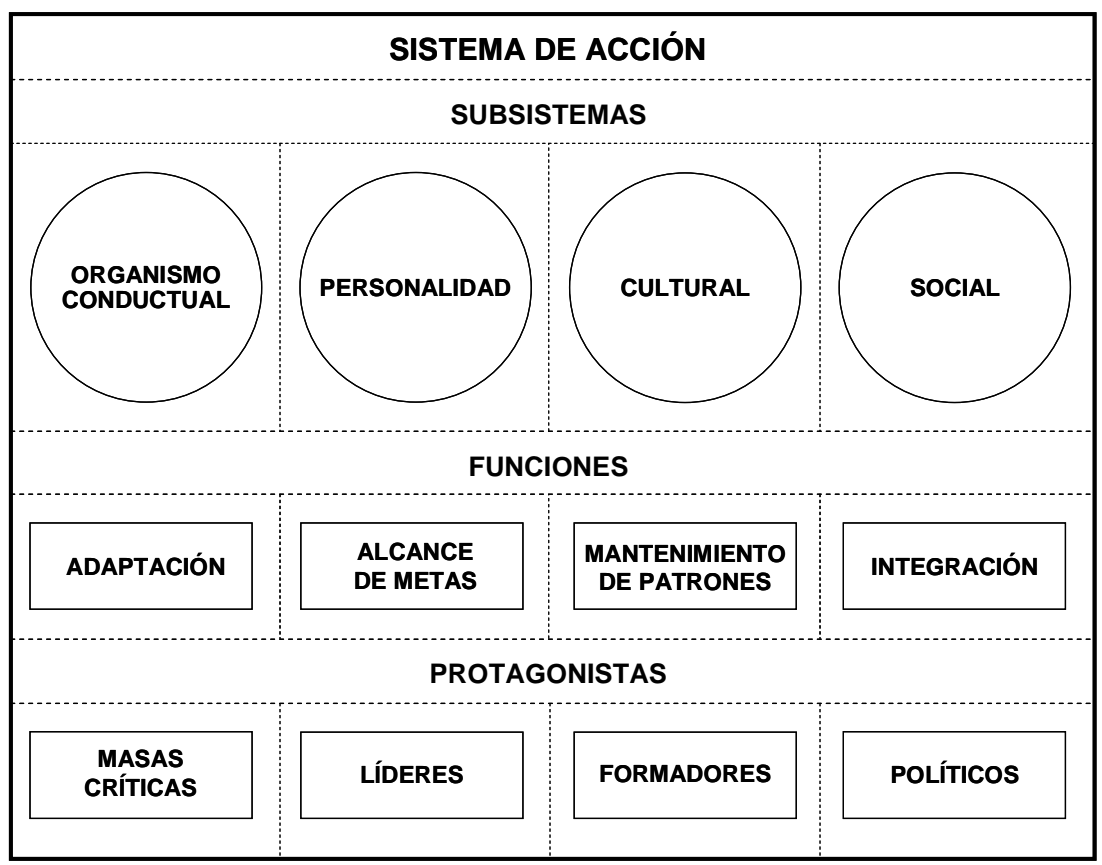

Figura 5. Principales componentes, funciones y actores de un sistema de acción.

\section{Competencias de los sistemas de acción}

En principio, una actividad elemental, pero muy compleja, de las personas que participan en el funcionamiento de un sistema de acción, es la adaptación individual y colectiva a los procesos de cambio, cualquiera que sea el origen y la naturaleza de éste. Más aún, cabe precisar, las competencias implicadas en la función de adaptación responden a un doble cometido: a) saber cómo adaptarse al cambio, y b) saber cómo generarlo; para ello, el sistema de acción requiere de personas y grupos que contribuyan al desarrollo del conocimiento (científico, tecnológico, artístico y humanista), como elemento esencial de adaptación. Por consiguiente, la solución de problemas y necesidades relacionados con la función de adaptación en un sistema de acción específico, requiere de cuadros de profesionales y expertos en distintas áreas de la ciencia, la tecnología, el arte y las humanidades, que promuevan los procesos de adaptación, desde ambas perspectivas y de manera equilibrada; asimismo, esos cuadros constituyen las masas críticas de los sistemas de acción para la gestión del cambio y la adaptación a éste. 
El alcance de metas, la segunda función sustantiva de los sistemas de acción, concierne particularmente a los líderes y las actividades éstos. Son estas personas quienes deben reunir una serie de competencias y cualidades de reconocido valor o mérito para fungir como autoridad o guía de un colectivo (grupo social, equipo de trabajo, empresa, organización, comunidad, pueblo, sociedad, país, región).

Por su parte, el mantenimiento de patrones representa la función crítica del subsistema cultural; función para la que son fundamentales las competencias de los formadores formales, no formales e informales-, en general, y de educadores e instructores profesionales, en particular, en lo relativo a la preservación, renovación y desarrollo del conocimiento -en materia de ciencias, tecnología, artes y humanidades-, entre los miembros del sistema de acción. Evidentemente, las funciones de adaptación y mantenimiento de patrones están estrechamente interrelacionadas, por lo que las actividades y competencias de sus principales actores deben complementarse, a sabiendas de que la disociación entre conocimientos y contextos es contraproducente para todo el sistema.

Finalmente, el subsistema social — que pudiera ser el primero- tiene por función principal la integración de los diversos componentes estructurales y funcionales del sistema de acción. Esta función está a cargo, en primera instancia, de los políticos, directivos y autoridades que gobiernan el sistema de acción, bajo un esquema de normas jurídicas y sociales.

\section{Pseudo-competencias y anti-competencias en los sistemas de acción}

Dada la importancia de las competencias implicadas en el funcionamiento y desarrollo de un sistema de acción, es necesario considerar la posibilidad de que dichas competencias no sean las adecuadas para atender de manera satisfactoria los problemas y necesidades del sistema mismo, e incluso, de que se haga mal uso de ellas, en menoscabo de la sustentabilidad y los intereses de todo el sistema y de otros sistemas. En tal caso, personas y grupos recurren a pseudo-competencias que, aunque no les confieren capacidades efectivas, sí les permiten guardar las apariencias y enmascarar deficiencias, en sus respectivas actividades y roles, al menos durante algún tiempo y bajo ciertas circunstancias. Las pseudo-competencias pueden clasificarse en cuatro grupos: 
a) "Competencias" de nombre — mal llamadas así.

b) "Competencias" aparentes o simuladas — que en realidad no se tienen o no se ejercen cabalmente.

c) "Competencias" huecas - carentes de competencia.

d) "Competencias" desnaturalizadas — corrompidas, deshonestas; anti-competencias.

El funcionamiento de los sistemas de acción puede verse afectado, en sentido positivo, por competencias individuales y colectivas apropiadas; o en sentido negativo, a falta de estas competencias, en determinado momento y lugar, para prevenir y resolver problemas y necesidades concretos.

En un sistema de acción, en tanto la naturaleza de los elementos primarios determina el carácter de las funciones primarias, son las competencias individuales y colectivas las que habilitan estas funciones; de ahí que, sin las competencias correspondientes, las funciones críticas se debilitan o desvirtúan, los elementos estructurales pierden su capacidad potencial, y los problemas del sistema de acción -en general- se incrementan y complican. Todo esto trae a colación, nuevamente, el tema de los aprendizajes centrales (capacidades nucleares, valores y principios) como soporte indispensable de competencias individuales y colectivas, y por lo tanto, también, de la estructura y funcionamiento de los sistemas de acción en que las personas viven y trabajan.

\section{CONCLUSIONES}

Integradas a la sociedad del conocimiento y los procesos de globalización, las modalidades dominantes de formación para la vida y el trabajo, tienen por directriz común el fomento de la competitividad de los bienes y servicios producidos, a través de sistemas estandarizados de certificación de las competencias individuales. Con ello, se tiende a perder de vista el papel de las competencias, tanto individuales como colectivas, en amplios contextos de la participación social y económica, de modo que el esfuerzo personal se concentra en intereses individuales y de grupos, y se desvincula de la concepción y solución de problemas en el ámbito colectivo y de terceros. 
Como productos e instrumentos de la formación de individuos y grupos, las competencias juegan un papel fundamental en la estructura y funcionamiento de los sistemas de acción en que la gente de una o más sociedades encuentra espacios de participación y desarrollo-; particularmente, en lo relativo a los principales elementos y funciones con que estos sistemas operan, y a los roles de masas críticas (de profesionales y expertos), líderes, formadores y autoridades y directivos. A los riesgos y contradicciones de las competencias desarticuladas, se suma el socorrido recurso de las pseudo-competencias, que de poco o nada valen para solucionar los complejos problemas y necesidades de los sistemas de acción, en el mundo actual.

Por las razones expuestas, es preciso que los valores de las personas se fomenten, no sólo en la esfera de las competencias —donde no pocas veces pasan inadvertidos—, sino más allá, por encima de lo que a éstas concierne, en el extenso ámbito de la educación y la cultura.

\section{REFERENCIAS}

Air Force Personnel Center. (s.f.). Managerial Competences. Recuperado el 14 de septiembre de 2004, de www.afpc.randolph.af.mil/cp/secccp/managerialcompetences.htm

Argüelles, Antonio (Comp.). (1996). Competencia laboral y educación basada en normas de competencia. México: Limusa.

Buckley, Finian, Monks, Kathy y McKevitt, Conor. (2002). Identifying management needs in a time of flux: A new model for human resource manager education. Dublín, Irlanda: Center for Management Learning and Development, Dublin City University Business School.

Burton, Lynn. (Ed.). (1992). Developing resourceful humans. Adult education within the economic context. Londres: Routledge.

Centre for Educational Research and Innovation - CERI. (1997). Prepared for life? How to measure cross-curricular competencies. París: OCDE.

(2000). Motivating students for

lifelong learning. París: OCDE.

CINTERFOR/OIT. (s.f.). Competencia laboral. Identificación y normalización de competencias. Recuperado el 9 de mayo de 2009, de 
http://www.ilo.org/public/spanish/region/ampro/cinterfor/temas/complab/observ/iii/mexic $\underline{\text { o/ii.htm }}$

Coles, Mike y Werquin, Patrick. (2007). Qualifications systems. Bridges to lifelong learning. París: OCDE.

Comisión Europea. (s.f., a). Glosario. Competencias. En: Elearningeuropa.info. Recuperado el 4 de abril de 2009, de http://www.elearningeuropa.info/main/index.php?lng=es\&page=glossary\&abc=C

. (s.f., b). Glossary. Skills. En: Elearningeuropa.info. Recuperado el 4 de abril de 2009, de http://www.elearningeuropa.info/main/index.php?page=glossary\&abc=S

Fernández-Salinero, Carolina. (2006). Las competencias en el marco de la convergencia europea: Un nuevo concepto para el diseño de programas educativos. Encounters on Education, 7, 131-153.

Gautier, Bénédicte y Vervisch, Marie-Odile. (1997). En: Psicología Laboral. Equipos de alto rendimiento. Recuperado el 15 de abril de 2009, de http://www.asimetcapacitacion.cl/alto rendimiento.htm

Girard, René. (2004). Definición de criterios y métodos para la caracterización, definición, selección y clasificación de competencias claves en México. Estudio de consultoría, ASISTEC, S.C. México, D.F.: Unidad Coordinadora y Administradora del PMETyC,

Gonczi, Andrew y Athanasou, James. (1996). Instrumentación de la educación basada en competencias. Perspectivas de la teoría a la práctica en Australia. En A. Argüelles (Comp.). Competencia laboral y educación basada en normas de competencia (pp. 265-288). México: Limusa.

Jokisch, Rodrigo. (s.f.). Talcott Parsons: Observaciones sobre su trabajo clásico 'La Teoría de la Estructura de la Acción Social' - desde el punto de vista de la Teoría de las Distinciones. Recuperado el 21 de julio de 2009, de http://www2.tuberlin.de/ society/Jokisch Parsons StructureOfSocialAction ES.htm\# ftn17

Kaplan, Robert y Norton, David. (2004). Strategy maps. Converting intangible assets into tangible outcomes. Boston, Massachusetts: Harvard Business School.

Lloyd, Chris y Cook, Amanda. (1993). Implementing standards of competence. Practical strategies for industry. Londres: Kogan Page.

Morfín, Antonio. (1996). La nueva modalidad educativa: educación basada en normas de competencia. En A. Argüelles (Comp.). La nueva modalidad educativa: educación basada en normas de competencia (pp. 81-98). México: Limusa.

Nyatanga, Lovemore; Forman, Dawn y Fox, Jane. (1998). Good practice in the accreditation of prior learning. Londres: Cassell Welligton House. 
OCDE. (1997). Prepared for life. How to measure cross-curricular competencies. Paris: OCDE.

. (2002). Definition and selection of competences (DeSeCo). Theoretical and conceptual foundations. Strategy paper. Recuperado el 10 de enero de 2008, de http://www.portal-stat.admin.ch/deseco/deseco strategy paper final.pdf

OIT. (1997). Formación basada en competencia laboral. Montevideo: Cinterfor/OIT.

Parsons, Talcott. (1974). El sistema de las sociedades modernas, México: Trillas.

Rodríguez, Nelson. (s.f.). ¿Qué son las competencias? En: CINTERFOR/OIT, Competencia Laboral. Recuperado el 10 de abril de 2009, de http://www.ilo.org/public/spanish/region/ampro/cinterfor/temas/complab/doc/otros/sel ef $\underline{\text { e/i.htm }}$

Salovey, Peter y Mayer, John. (1990). Emotional intelligence. Imagination, Cognition and Personality, 9 (3), 185-211.

Tuning Project. (2004). Tuning educational structures in Europe. Tuning methodology. Recuperado el 15 de febrero de 2009, de http://tuning.unideusto.org/tuningeu/index.php?option=content\&task=view\&id=172\&ltem $\underline{i d=205}$ 